\title{
Flory-Huggins Parameters for Thiol-ene Networks using Hansen Solubility Parameters
}

Nicholas J. Bongiardina ${ }^{1}$, Jasmine Sinha ${ }^{2}$, Christopher N. Bowman ${ }^{1,2, *}$

${ }^{1}$ Materials Science and Engineering Program, University of Colorado, Boulder, CO, 80309-0596 USA

${ }^{2}$ Department of Chemical and Biological Engineering, University of Colorado Boulder, CO80303, United States.

\section{Supporting Information}

Table of contents:

1. Dynamic mechanical analysis of thiol-ene networks $\quad \mathrm{S} 2$

2. Plot of $\delta_{\mathrm{h}}$ vs $\delta_{\mathrm{p}}$ for every solvent with categorization of "poor", "good", and "great" $\quad \mathrm{S} 2$

3. Hansen solubility parameters determined from HSPiP for the polymer networks and S3 solvents tested in swelling experiments

4. Swelling ratio, measured Flory-Huggins Parameter and predicted Flory-Huggins S4 Parameter using HSPiP 


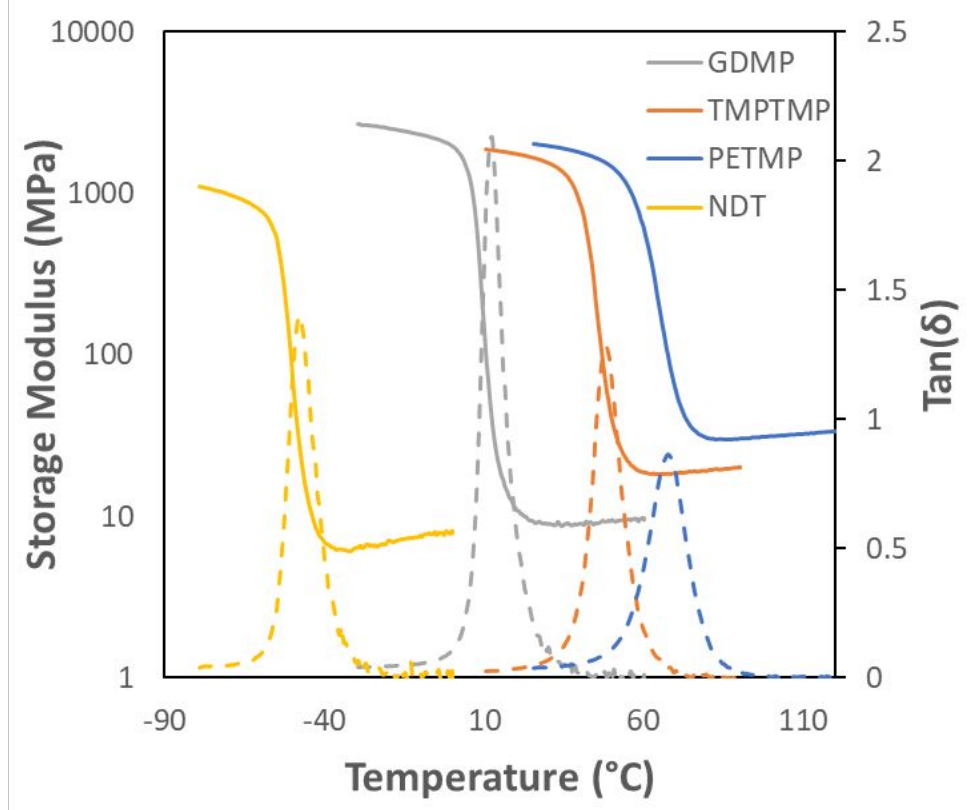

Figure S1: Dynamic mechanical analysis for each of the three thiol-ene networks after the sol-fraction has been removed via swelling in acetone. The storage modulus at $\mathrm{T}_{\mathrm{g}}+40$ was used to calculate the Flory-Huggins interaction parameter. The molecular weight between crosslinks was determined for each network to be: PETMP - 350 g/mol, TMPTMP - 540 g/mol, GDMP - 1020 g/mol, NDT, 1020 $\mathrm{g} / \mathrm{mol}$.

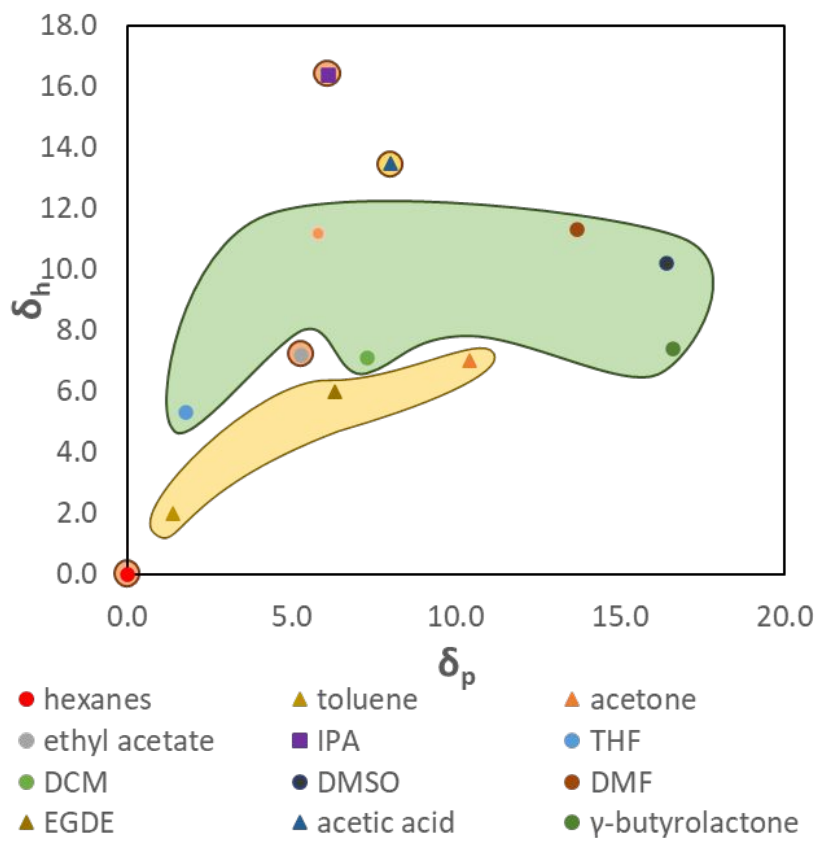

Figure S2: Plot of the hydrogen bonding versus the polar Hansen solubility parameter. The solvents categorized as "great" are highlighted in green, "good" are highlighted in yellow, and "poor' are highlighted in red. 
Table S1: Hansen solubility parameters determined from HSPiP for the polymer networks and solvents tested in swelling experiments.

\begin{tabular}{|l|rrr|}
\hline & \multicolumn{1}{|c|}{$\delta_{\mathrm{d}}$} & \multicolumn{1}{c|}{$\delta_{\mathrm{p}}$} & \multicolumn{1}{c|}{$\delta_{\mathrm{h}}$} \\
\hline PETMP-TTT & 18.6 & 8.4 & 4.1 \\
TMPTMP-TTT & 18.6 & 8.5 & 4.2 \\
GDMP-TTT & 18.7 & 8.7 & 4.3 \\
NDT-TMPTE & 18.4 & 2.4 & 2 \\
\hline Hexanes & 14.9 & 0 & 0 \\
toluene & 18 & 1.4 & 2 \\
acetone & 15.5 & 10.4 & 7 \\
ethyl acetate & 15.8 & 5.3 & 7.2 \\
IPA & 15.8 & 6.1 & 16.4 \\
THF & 16.8 & 5.7 & 8 \\
DCM & 17 & 7.3 & 7.1 \\
DMSO & 18.4 & 16.4 & 10.2 \\
DMF & 17.4 & 13.7 & 11.3 \\
EGDE & 15.4 & 6.3 & 6 \\
acetic acid & 14.5 & 8 & 13.5 \\
Y-butyrolactone & 18 & 16.6 & 7.4 \\
Aniline & 20.1 & 5.8 & 11.2 \\
\hline
\end{tabular}


Table S2: Swelling ratio ( $\lambda$, the Flory-Huggins Parameter $\left(\chi_{\mathrm{m}}, \mathrm{MPa}^{0.5}\right)$, measured swelling experiments, and FloryHuggins parameter predicted by $\operatorname{HSPiP}\left(\chi_{\mathrm{p}}, \mathrm{MPa}^{0.5}\right)$ for the three polar networks made PETMP, TMPTMP, and GDMP with TTT. A red X (X) denotes that the network did not swell in that solvent. All errors represented are the standard deviation. *only one sample survived swelling, no standard deviation possible.

\begin{tabular}{|l|c|c|c|c|c|c|c|c|c|}
\hline \multirow{2}{*}{ Solvent } & \multicolumn{3}{|c|}{ PETMP-TTT } & \multicolumn{3}{c|}{ TMPTMP-TTT } & \multicolumn{3}{c|}{ GDMP-TTT } \\
\cline { 2 - 9 } & $\lambda$ & $x_{\mathrm{m}}$ & $\mathrm{x}_{\mathrm{p}}$ & $\lambda$ & $\mathrm{x}_{\mathrm{m}}$ & $\chi_{\mathrm{p}}$ & $\lambda$ & $\chi_{\mathrm{m}}$ & $\chi_{\mathrm{p}}$ \\
\hline hexanes & $\mathrm{X}$ & $\mathrm{X}$ & 1.1 & $\mathrm{X}$ & $\mathrm{X}$ & 1.1 & $\mathrm{X}$ & $\mathrm{X}$ & 1.2 \\
\hline toluene & $\mathrm{X}$ & $\mathrm{X}$ & 0.35 & $0.16 \pm 0.04$ & $1.0 \pm 0.1$ & 0.36 & $0.223 \pm 0.003$ & $0.971 \pm 0.006$ & 0.39 \\
\hline acetone & $0.156 \pm 0.002$ & $0.931 \pm 0.007$ & 0.22 & $0.19 \pm 0.02$ & $0.95 \pm 0.07$ & 0.22 & $0.288 \pm 0.008$ & $0.86 \pm 0.01$ & 0.23 \\
\hline ethyl acetate & $0.068 \pm 0.009$ & $1.4 \pm 0.1$ & 0.30 & $0.21 \pm 0.01$ & $0.89 \pm .02$ & 0.30 & $0.315 \pm 0.005$ & $0.833 \pm 0.008$ & 0.32 \\
\hline IPA & $\mathrm{X}$ & $\mathrm{X}$ & 0.87 & $\mathrm{X}$ & $\mathrm{X}$ & 0.86 & $0.030 \pm 0.006$ & $2.29 \pm 0.2$ & 0.86 \\
\hline THF & $0.255 \pm 0.008$ & $0.69 \pm 0.02$ & 0.26 & $0.36 \pm 0.004$ & $0.66 \pm 0.01$ & 0.26 & $0.39 \pm 0.04$ & $0.78 \pm 0.05$ & 0.28 \\
\hline DCM & $0.303 \pm 0.005$ & $0.91 \pm 0.01$ & 0.08 & $0.32 \pm 0.07$ & $1.01 \pm 0.1$ & 0.07 & $0.39 \pm 0.06$ & $1.00 \pm 0.07$ & 0.08 \\
\hline DMSO & $0.36 \pm 0.01$ & $0.58 \pm 0.02$ & 0.50 & $0.400 \pm 0.003$ & $0.690 \pm 0.005$ & 0.49 & $0.67 \pm 0.02$ & $0.62 \pm 0.01$ & 0.46 \\
\hline DMF & $0.316 \pm 0.006$ & $0.59 \pm 0.01$ & 0.40 & $0.37 \pm 0.02$ & $0.67 \pm 0.03$ & 0.39 & $0.62 \pm 0.05$ & $0.59 \pm 0.03$ & 0.38 \\
\hline EGDE & $0.15 \pm 0.01$ & $0.86 \pm .04$ & 0.31 & $0.217 \pm 0.001$ & $0.84 \pm 0.003$ & 0.31 & $0.21 \pm 0.02$ & $1.00 \pm 0.04$ & 0.33 \\
\hline acetic acid & $0.149 \pm 0.02$ & $1.2 \pm 0.1$ & 0.54 & $0.231 \pm 0,003$ & $1.05 \pm 0.01$ & 0.54 & $0.33 \pm 0.01$ & $0.96 \pm 0.01$ & 0.54 \\
\hline GBL & $0.36 \pm 0.02$ & $0.63 \pm 0.04$ & 0.37 & $0.470 \pm .002$ & $0.64 \pm 0.002$ & 0.36 & $1.08 \pm 0.01$ & $0.42 \pm 0.006$ & 0.34 \\
\hline aniline & $0.41 *$ & $0.37 *$ & 0.37 & $0.70 \pm .01$ & $0.27 \pm .01$ & 0.36 & $1.18 \pm 0.06$ & $0.25 \pm 0.03$ & 0.35 \\
\hline
\end{tabular}

Table S3: Swelling ratio $(\lambda$, the Flory-Huggins Parameter $\left(\chi_{\mathrm{m}}, \mathrm{MPa}^{0.5}\right)$, measured swelling experiments, and Flory-Huggins parameter predicted by HSPiP $\left(\chi_{\mathrm{p}}, \mathrm{MPa}^{0.5}\right)$, for the nonpolar network NDT-TMPTAE. All errors represented are the standard deviation.

\begin{tabular}{|l|c|c|c|}
\hline \multirow{2}{*}{ Solvent } & \multicolumn{3}{|c|}{ NDT-TMPTAE } \\
\cline { 2 - 4 } & $\lambda$ & $\chi_{\mathrm{m}}$ & $\chi_{\mathrm{p}}$ \\
\hline hexanes & $0.14 \pm 0.01$ & $1.05 \pm 0.05$ & 0.47 \\
\hline acetone & $0.14 \pm 0.02$ & $1.23 \pm 0.07$ & 0.54 \\
\hline DMSO & $0.044 \pm 0.009$ & $2.3 \pm 0.2$ & 1.30 \\
\hline acetic acid & $0.155 \pm 0.004$ & $1.36 \pm 0.01$ & 0.57 \\
\hline GBL & $0.086 \pm 0.02$ & $1.76 \pm 0.01$ & 1.07 \\
\hline
\end{tabular}

